\title{
Relativity and the Causal Efficacy of Abstract Objects
}

\section{Tim Juvshik}

\author{
Penultimate Draft, please cite the published version (American Philosophical Quarterly, \\ forthcoming)
}

\begin{abstract}
Abstract objects are standardly taken to be causally inert, however principled arguments for this claim are rarely given. As a result, a number of recent authors have claimed that abstract objects are causally efficacious. These authors take abstracta to be temporally located in order to enter into causal relations but lack a spatial location. In this paper, I argue that such a position is untenable by showing first that causation requires its relata to have a temporal location, but second, that if an entity is temporally located then it is spatiotemporally located since this follows from the theory of Relativity. Since abstract objects lack a spatiotemporal location, then if something is causally efficacious, it is not abstract.
\end{abstract}

\section{Introduction}

Abstract objects are standardly taken to be causally inert. This assumption is ubiquitous in philosophy, both within metaphysics and elsewhere. However, explicit arguments for the causal inertness of abstract objects are rarely given. ${ }^{1}$ As a result, an increasing number of philosophers have claimed that abstract objects can enter into causal relations. For example, both Julian Dodd (2007, 2012) and Ben Caplan and Carl Matheson (2004) argue that abstract types can enter into causal relations in virtue of derivatively participating in events. Stuart Brock, Cei Maslen, and Justin Ngai (2013) argue that there are no good arguments in support of the causal inertness of abstracta, so the claim can be straightforwardly denied. John Burgess and Gideon Rosen (1997, pp. 23-25) take certain abstracta, including impure sets, properties, novels, and fictional characters to be both causally active and causally acted upon. Benjamin Callard (2007, pp. 355) argues that "there is no problem with the idea of abstract objects effecting changes in us; there is no conceptual difficulty with the idea that they impart energy to our brains". ${ }^{2}$ 
The rejection of the standard view of abstracta as causally inert is usually motivated by one of three reasons. First, the causal theory of reference developed by Kripke and Putnam requires standing in causal relations to the referents of our terms. If abstract entities are causally inert then it's unclear how we can refer to them. ${ }^{3}$ Second, as is well-known, Benacerraf's problem takes it be a mystery how we can have mathematical knowledge if mathematical entities, the paradigm cases of abstract objects, don't enter into causal relations. ${ }^{4}$ Third, a common position in the philosophy of art is that repeatable artworks - those artworks that can have multiple instances like music, film, and literature - are abstract objects, and fictional characters are similarly abstract. However, we also commonly think that we can hear, see, or read such works, and that authors literally create fictional characters and works of art. $^{5}$ None of this looks to be possible if abstract objects are causally inert, and thus there are strong motivations for denying the standard view.

One thing common to these proposals is that they take abstract entities to have a temporal location, but no spatial location. Thus, Dodd writes that "types are abstract entities, which, as I use the term, means that they have no location in space" (2007, pp. 12). Similarly, Brock, Maslen and Ngai (2013, pp. 68) take a lack of spatial location to be necessary for being abstract, while still allowing that they have temporal location. Burgess and Rosen (1997, pp. 20-21) take most abstract entities, including numbers, to exist in time but 'outside' of space, while Callard takes the Platonist position to be that "the objects of mathematics inhabit a necessary and unchanging realm of nonspatial eternal entities" (2007, pp. 347). Caplan and Matheson (2004, pp. 120) likewise seem to endorse the non-spatial criterion for certain abstracta like fictional characters and musical works. ${ }^{6}$ Thus, these authors are committed to the following three theses (although they differ on which abstracta they apply to): (i) abstract objects lack a spatial location; (ii) abstract objects have a temporal location; and (iii) abstract objects are causally efficacious. In this paper, I present an 
argument for the causal inertness of abstract objects by showing that the above position is untenable. I do this by showing that something can't be causally efficacious without being temporally located, but that something can't have a temporal location without a spatial location.

The argument - which I call the argument from Relativity ${ }^{7}$ - is in the form of a hypothetical syllogism, as follows:

(P1) If $\mathrm{x}$ is causally efficacious, then $\mathrm{x}$ has a temporal location.

(P2) If $\mathrm{x}$ has a temporal location, then $\mathrm{x}$ has a spatiotemporal location.

(P3) If $\mathrm{x}$ has a spatiotemporal location, then $\mathrm{x}$ is not abstract.

(C) So, if $\mathrm{x}$ is causally efficacious, then $\mathrm{x}$ is not abstract.

By "causally efficacious" I mean that $\mathrm{x}$ can be a relatum of the causal relation (i.e. a potential cause or effect). The argument shows that causal inertness follows from a lack of spatiotemporal location. (P1) is, I will argue, a conceptual truth about causation. (P2) follows from the theory of Relativity, both Special and General. (P3) is a result of the standard way of making the abstract/concrete distinction. (C) follows straightforwardly on this characterization of the abstract/concrete distinction.

Those who deny the causal inertness of abstract objects tend to accept (P1), since they attribute temporal location to abstracta so they can enter into causal relations. They also accept a modified version of (P3), taking abstract objects to lack a spatial location. Therefore, what they deny is the inference in (P2): they think that abstract objects can have temporal location without spatial location.

The paper is structured as follows. In section 2 I adopt and discuss the standard characterization of the abstract/concrete distinction, which Lewis calls the Way of Negation, and suggest ways it can handle putative counterexamples. In section 3 I establish (P1): I argue that 
causal efficacy entails having a temporal location, since this is a conceptual truth about causation. In section $4 \mathrm{I}$ defend (P2) by arguing that having a temporal location entails having a spatiotemporal location, since our best physical theories, in particular the theory of Relativity, takes the universe to be a fundamentally unified four-dimensional manifold. There are no independent spatial or temporal relations, only spatiotemporal relations. Since having a spatiotemporal location is incompatible with being abstract, it follows that being causally efficacious entails not being abstract. Finally, in section 5 I consider several objections to the argument and offer replies, before concluding in section 6.

Before proceeding, I want to note the following three assumptions that I make in this paper. First, I will assume some version of the B-theory of time: the view that all times, past, present, and future, are all equally real. Second, I will not assume any particular account of causation such as, e.g. the counterfactual theory or the energy transfer view or production accounts, etc. The argument from Relativity will apply to any analysis of causation. Third, I will assume something about causal relata, namely, that objects, properties and events can all enter into causal relations. While we frequently treat events as the primary relata of causal relations, we also speak of objects as causes and effects (e.g. 'the baseball broke the window'), as well as property instances (e.g. 'the humidity of the air corroded the metal'). ${ }^{8}$ I will remain neutral on whether such talk should be understood as objects and properties being causes and effects directly, or indirectly in virtue of participating in events. ${ }^{9}$

\section{Establishing P3: The Way of Negation}

A number of ways of characterizing the abstract/concrete distinction have been offered, a classic discussion of which is by David Lewis (1986, pp. 81-86). ${ }^{10}$ By far the standard view, which I think has claim to default status, is what Lewis calls the Way of Negation - that abstracta lack a 
spatiotemporal location and are causally inert, while concreta are those entities that have a location in spacetime and are causally efficacious. ${ }^{11}$ So as not to beg any questions, I will understand the Way of Negation as treating abstracta as lacking a spatiotemporal location while concreta are spatiotemporally located. Indeed, the main argument of this paper is that causal inertness follows from a lack of spatiotemporal location. We therefore get the following biconditional:

The Way of Negation: $\mathrm{x}$ is abstract iff $\mathrm{x}$ lacks a spatiotemporal location.

Lacking a spatiotemporal location is both necessary and sufficient for being abstract, and it's highly intuitive for the paradigmatic cases of abstract entities: numbers, propositions, properties, and sets all prima facie seem not to be located in spacetime. Indeed, it seems like a category mistake to ask where the number two or the null set or the proposition that there are no penguins in the Arctic are located. If one worries that spacetime points will turn out to be abstract, then we can say that they are trivially self-located. The assumption that abstracta lack a spatiotemporal location is pervasive. ${ }^{12}$ Even those who reject causal inertness adopt some version of it.

Nonetheless, there are some putative abstracta that seem to be counterexamples to the Way of Negation. These typically target its ability to provide necessary conditions for being abstract. Impure sets are usually thought to be located where their members are and abstract artifacts like musical works or fictional characters are thought to be created at particular times so are temporally located. Since these entities are usually thought to be abstract, it might be argued that lacking a spatiotemporal location isn't necessary for being abstract. I want to suggest various ways one could handle these counterexamples and thereby show that lack of spatiotemporal location adequately characterizes the abstract/concrete distinction. ${ }^{13}$

Let's look at each of these potential counterexamples in turn. Most philosophers assume that sets, including impure sets, are abstract. However, many claim that it's intuitive that the 
singleton set containing Socrates is located wherever Socrates is located. How might we respond to this alleged counterexample? We might take one of three routes. First, following Penelope Maddy (1990), we might argue that sets aren't a unified ontological category. Pure sets are abstract since they aren't spatiotemporally located while impure sets are concrete and are located wherever their members are. However, the abstract/concrete distinction is standardly thought to exhibit certain formal features that any account of it should accommodate (see Cowling 2017, pp. 70-72 for discussion of these features). In particular, the distinction is generally thought to be exclusive, i.e. nothing can be both abstract and concrete. This is because abstract and concrete are thought to be contradictory, joint-carving ontological categories. ${ }^{14}$ Maddy's view seems to reject the exclusivity condition, since sets containing both abstract and concrete members will presumably be both abstract and concrete. ${ }^{15}$ Perhaps one could claim that the ontological category of one member of a set is 'contagious': the set containing me and the number four is abstract because abstracta 'contaminate' the ontological category of the set (or alternatively concreta take precedence). This would be a way of retaining exclusiveness on Maddy's proposal.

Second, we might accept that impure sets are abstract, but deny that they are located where their members are. Thus, all sets, pure and impure, are transcendent in the same way that transcendent universals are: they don't have a spacetime location, but exist in some Platonic heaven. In the case of $\{$ Socrates $\}$, the set exists independently of Socrates himself. This view has been defended by Lowe (1995, pp. 523-4), ${ }^{16}$ but it has the downside of rejecting the extensional equivalence of sets and their members.

Third, we can adopt the view defended by Max Black (1971) whereby sets are nothing but plural referring expressions. On Black’s view, the impure set \{Tom, Dick, Harry $\}$ is just a way to plurally refer to Tom, Dick, and Harry. Philosophers mistakenly reify set-talk, so think that there 
must be some entity that is the set $\{$ Tom, Dick, Harry $\}$. On such a view, singleton sets are singular referring expressions while the null set is an expression that fails to refer. Since sets aren't entities on Black's view, it doesn't make sense to ask where they're located. I'm not sure which of these views to adopt, but any one of them will avoid the putative counterexample.

With respect to the case of abstract artifacts, there are two options. First, one can accept that there are such entities, and that they are created and destroyed, but deny that such creation and destruction entails that they have a temporal location. This seems to be Amie Thomasson's (1999) position, whereby abstract artifacts come into existence in virtue of relations of ontological dependence on concreta, so a novel comes into existence with its first copy. Importantly on Thomasson's account, having a temporal origin doesn't entail that the resulting entities have spatiotemporal locations. Thus, while a novel, say, comes into existence in virtue of various creative acts by its author that occur at specific times, the novel itself doesn't have a location in time.

Second, one can accept that there are such entities but deny that they are created, but rather are creatively discovered. Julian Dodd (2007) defends this view with respect to musical works, while Jerrold Katz (1998, pp. 132-138) defends it for abstract artifacts generally, including games and languages. On this view, authors and composers discover atemporal novels or musical works just as Marie Curie discovered radium. The discovery occurs at a specific time, but the entity discovered doesn't have a temporal location. Again, I'm undecided between which route is preferable, but one of these accounts allows for abstract artifacts to lack a spatiotemporal location.

Therefore, the putative counterexamples can be resisted. Not only does the Way of Negation fit well with paradigmatic abstracta, it also has claim to being the standard or default view in the literature, so has a concomitantly broad appeal. More importantly, since those who 
defend the causal efficacy of abstract objects accept the Way of Negation, at least in part, I will assume it for present purposes. I return to the issue of only adopting the non-spatial condition in section 4.

\section{Establishing P1: Causation and Time}

It's plausible that for $\mathrm{C}$ to cause $\mathrm{E}$ both $\mathrm{C}$ and $\mathrm{E}$ must have some (perhaps definite) temporal location. When we say something like 'the boiling water scalded her' it seems that the two events - the boiling of the water and her scalding - exist at (distinct) times. This also holds for property instances and objects: the drink's bitterness causes me to wince, and the drink is bitter for a particular duration (its entire existence, say); similarly, that the baseball broke the window requires that the baseball and window have a temporal location in order to be related as cause to effect. Perhaps these last two cases can be understood as properties and objects participating in events, perhaps not - it doesn't matter, since either way they all have temporal location.

The claims that causes must precede their effects and that there is no backwards causation presuppose that causation is inherently temporal. Even if there is simultaneous or backwards causation, the causal relata still have temporal locations (i.e. bear temporal relations to one another), since simultaneity just means occurring at the same time and backwards causation means the cause must produce its effect at an earlier time than itself. Regular causal statements of the form ' $\mathrm{x}$ caused $\mathrm{y}$ ' that assume the temporal priority of the cause assert a temporal relation, which in turn entails temporal locations for its relata. For $\mathrm{x}$ to cause $\mathrm{y}$, both $\mathrm{x}$ and $\mathrm{y}$ need to be temporally related and therefore temporally located; all of our familiar, everyday causal claims relate entities that exist in time. On the face of it, it seems to be a conceptual or a priori truth that having temporal location is necessary for being causally efficacious. For something to be a cause of something else 
just is for it to exist at an earlier time. ${ }^{17}$ Therefore, for something to be a cause or effect it must be located in time.

While the above might seem relatively quick, (P1) is accepted by those who defend the causal efficacy of abstracta, including Dodd (2007), Brock, Maslen and Ngai (2013), Caplan and Matheson (2004), Burgess and Rosen (1997), and Callard (2007). It's therefore uncontroversial in the present context.

\section{Establishing P2: Relativity, Time, and Spacetime}

The various authors who defend the causal efficacy of abstract objects reject (P2), that having a temporal location entails having a spatiotemporal location. That is, they accept (P1), taking causation to require temporal location, and thus take abstract objects to have a temporal location. It might be thought that they also reject (P3), that abstracta lack a spatiotemporal location. This is partly true: they accept a version of (P3) because they take abstract objects to have a temporal location but lack a spatial location. They adopt the non-spatial condition in order to give some characterization of what it is to be abstract, even if it only provides a necessary, but not sufficient condition. As I mentioned earlier, Dodd (2007, ch. 3) explicitly adopts the lack of spatial location as the defining feature of abstracta, arguing that they exist eternally rather than atemporally, since he wants to be able to say that we hear a musical work, which is a causal process. Similarly, Brock, Maslen, and Ngai (2013) adopt the lack of spatial location as a necessary condition on being abstract so that fictional characters lack a spatial location but are created (which they take to be causal) at specific times, and thus have temporal location. Callard (2007, pp. 347, 352) also takes mathematical entities to be non-spatial but exist eternally rather than atemporally. Thus, they reject the inference in (P2): an entity can have temporal location without spatiotemporal location, and this is precisely how they characterize abstract objects. However, if (P2) is true, as I will argue in 
this section that it is, then such a position is untenable. Dodd, Callard and friends become forced to accept the full Way of Negation, whereby abstracta lack a spatiotemporal location, and as a result, are causally inert.

Newtonian spacetime takes there to be an independent spatial metric, which provides spatial distances between things, and an independent temporal metric, which provides temporal distances between things. As a result, in Newtonian spacetime it is intelligible to maintain that a class of things can bear merely spatial or merely temporal relations to each other. For example, two things or events could be separated in time, and thereby have temporal locations, but not be separated in space, and thereby lack spatial locations, or vice versa.

In Lorentzian spacetime there is only a single, spatiotemporal metric, which yields spatiotemporal distances (or the spacetime interval) between things or events. Lorentzian spacetime is the spacetime of our best physical theories - the theories of Special and General Relativity. ${ }^{18}$ In Lorentzian spacetime there is no purely spatial or purely temporal metric, and thus things or events cannot bear merely spatial or merely temporal relations to each other.

As a result, in Lorentzian spacetime it's not possible to make the move described above: abstracta can't have a temporal location but no spatial location. One cannot say that some class of things is temporally located but not spatially located (i.e. that they bear temporal relations to each other but not spatial relations) - there aren't any such relations to appeal to. In Lorentzian spacetime there's only one kind of relation, spatiotemporal relations, which things either bear or don't bear to each other. ${ }^{19}$

Since Lorentzian spacetime precludes the possibility of something only having a temporal location, this raises the following question: how are we to understand the claim that something is 'temporally located'? We already saw that it can't mean that the thing only bears temporal 
relations, since there are no such relations. There are two ways to go here. First, we could take such talk to be unintelligible or false. That is, we could say that it simply makes no sense to say that something has temporal location without spatial location in the context of Lorentzian spacetime or we could take such claims to be straightforwardly false. A second, more charitable reading would be to understand such talk as implicitly ascribing the only temporal-like relations that exist in Lorentzian spacetime, namely spatiotemporal relations. Since this second reading doesn't dismiss such claims out of hand, this is how I will understand such talk. But what follows from this is that if an entity has 'temporal location', then it has spatiotemporal location, which is (P2).

A structurally similar point is made by Robert Weingard (1977) and Michael Lockwood (1989) in the context of the identity theory of mind. One argument against reductively identifying the mental and the physical was the claim that while the physical is essentially spatial, temporal, and causal, the mental was essentially temporal and causal but non-spatial. Thus, they couldn't be identical. Weingard and Lockwood respond to this argument with a similar appeal to the theory of Relativity and the spacetime interval. If the mental is located in time, then it is located in spacetime. Weingard and Lockwood then conclude that the mental must be spatially located, as well, where 'having spatial and temporal location' is the pre-relativistic way of saying 'having spatiotemporal location'. Phil Dowe (2009) makes a similar argument against causation by absence, arguing that if absences are causes and effects, then, according to Relativity, they must be spatiotemporally located - effects must be located in the forward lightcone of their causes - but Dowe argues that there is no plausible location in which to locate them that doesn't entail that they are space-like separated from their causes. 
As a result, we've established (P2), having temporal location entails having spatiotemporal location. If abstracta are temporally located in order to be causally efficacious, then they are spatiotemporally located. I have already established (P1) and (P3), and the argument is valid. For these reasons, I conclude that if something is causally efficacious then it is not abstract.

\section{Objections and Replies}

In this section I consider four objections to the argument from Relativity and offer replies.

\subsection{Deny Causation Requires Time}

One might reject (P1) by claiming that certain accounts of causation don't require time. Sam Baron and Kristie Miller (2015) have recently argued that a counterfactual theory of causation doesn't require time by appeal to cases of counterfactual dependence in quantum mechanics. Baron and Miller assume both a counterfactual theory of causation and a B-theory of time. They argue that, given a case of two entangled particles, there is a possible world that is an intrinsic duplicate of this state of affairs but which lacks a time dimension; it only has a spatial manifold in which all events occur (where events are understood merely spatially in this world). Baron and Miller (2015, pp. 31-34) argue that all the same counterfactual dependencies are supported in this world and thus on the counterfactual theory of causation there is causation in this world but no time.

Two things are worth noting about Baron and Miller's view. First, it's not clear that their argument helps secure atemporal causation by abstracta because the example of counterfactual dependency between entangled particles requires them to have a spatial location. But at least in the actual world (and all nomically possible worlds), having a location in space will entail having a location in time, so while abstracta may be atemporal causes in timeless worlds, it's not clear how they could avoid being temporally located in the actual world. If we can't secure their causal efficacy in the actual world, then there's little to be gained by arguing for the possibility of their 
causal activity in other worlds, since abstracta won't be able to satisfy the causal role required for reference, knowledge, and artistic practice at the actual world that motivated the view in the first place.

Second, the counterfactual theory of causation has independent problems with abstract objects. Necessary existence is commonly taken to be a necessary feature of the most paradigmatically abstract entities such as numbers, propositions, and properties. However, if some or all abstract entities exist necessarily, then they seem to vacuously satisfy the counterfactual conditionals that characterize causation. That is, the counterfactual theory of causation ${ }^{20}$ takes $^{-1}$ causation to be analyzable in terms of counterfactual dependence, such that $\mathrm{C}$ is a cause of $\mathrm{E}$ iff

(i) If C were to occur, then E would occur, and

(ii) If $\mathrm{C}$ were not to occur, then $\mathrm{E}$ would not occur

For example, that the lightning strike caused the thunder satisfies both (i) and (ii), since if the lightning strike occurs, then the thunder occurs, and if the lightning hadn't struck, then the thunder wouldn't have occurred. While counterfactual dependence is now treated as sufficient but not necessary for causation, it still poses a problem for entities that exist necessarily. For example, we have the highly unintuitive consequence that the number 4 caused me to get out of bed this morning. If the number 4 exists necessarily, then for any value of $E$ the number 4 will count as a cause of E since in no worlds will 4 fail to exist, so the antecedent of (ii) will always be false, so the counterfactual will always be true. That is, necessarily existing abstract entities will all vacuously satisfy (ii).

Thus, on the counterfactual theory of causation, if abstracta can be causes, then we have a proliferation of spurious causes, since all necessarily existing abstract entities will be the cause of everything concrete and abstract (but no concreta will ever be the cause of any abstracta). Since 
Baron and Miller's argument crucially relies on the counterfactual theory of causation, their account will vastly overgenerate causation by abstracta. Thus, not only can we resist Baron and Miller's argument for atemporal causation, we also have an independent reason not to take abstract objects to be causes if the counterfactual theory of causation is true. ${ }^{21}$

\subsection{Abstracta are Non-Located Constituents of Events}

A second objection to (P1) might take events to be the primary causal relata and argue that abstracta are non-located constituents of events, and thereby causally efficacious in some 'derivative' sense. That is, one might argue that redness in some sense constitutes the event of my seeing a red apple, and thus can be said to be a derivative 'cause' of my visual experience in virtue of the apple's instantiation of redness or that the number two is in some sense involved in a twocar pile-up. This rejects (P1) by saying that there is a sense in which something can be a cause without being temporally located.

While there is something intuitively appealing about the claim that redness is in some sense involved in my experience of a red apple, this view has two major problems. First, in what sense do abstracta constitute events? Either the constitution relation being invoked is mereological or it isn't. If it's mereological, then abstracta are parts of concrete events. However, this violates the exclusivity condition I mentioned earlier: an entity cannot be both abstract and concrete. But an entity that has both abstract and concrete objects as parts seems to violate exclusivity, since presumably it would be both abstract and concrete. On the other hand, if the constitution relation invoked is not mereological, then it's unclear in what sense abstracta are 'constituents' of events. More would need to be said about this view to make it feasible.

Second, I have recently discussed at length this sort of 'derivative' causation by abstracta (Juvshik 2018). I argue that such a view entails widespread and systematic overdetermination. This 
is because both the concrete instantiation of redness and the abstract universal redness are sufficient causes of my visual experience of seeing a red apple. Since such overdetermination is prima facie undesirable, this view is equally unpalatable. Due to the mereological concern and the problem of overdetermination that I've previously addressed, I will set this objection aside.

\subsection{Instantiation is Causation}

Another response would be to claim that the instantiation relation is a causal relation, which would also be a counterexample to (P1), since at least one relatum doesn't have temporal location. ${ }^{22}$ But this response runs counter to current views, where instantiation is generally thought to be a kind of non-causal metaphysical dependence. I find it difficult to understand how one entity, which exists outside of spacetime, can "cause" a spatiotemporally located one. This picture seems to run into issues of systematic overdetermination, which I've recently discussed (Juvshik 2018). If instantiation is causation, then any property instance will be caused by both a chain of concrete events and the universal "causing" its instantiation, with such overdetermination looking undesirable.

Even granting that instantiation is a causal relation, it seems importantly different in kind. Following Aristotle, one could call abstract objects "formal" causes insofar as they are instantiated in objects. ${ }^{23}$ While such a view isn't implausible, the argument from Relativity is concerned with whether abstracta are Aristotelian efficient causes - whether they're the types of entities that we can bump into or hear, see, or create - which corresponds to our everyday concept of cause. Even so, I prefer to think of Aristotelian formal causes as a variety of non-causal explanation, and thus such an account wouldn't be attributing causal efficacy to abstracta in any sense. Indeed, cause and effect are usually taken to be distinct, and the instantiation of a universal and the universal itself don't appear to pass such a test. ${ }^{24}$ As a result, this response can be rejected. 


\subsection{The Way of Occupation}

A third objection comes from Sam Cowling and Wesley Cray (2017), who develop a version of the Way of Negation called the Way of Occupation. While their concerns are primarily with the omnipresence of various deities, they suggest that the account can be extended to abstract entities. Cowling and Cray characterize this way as follows:

The Way of Occupation: $\mathrm{x}$ is an abstract entity only if there is no spatiotemporal region $\mathrm{r}$ such that $\mathrm{x}$ occupies $\mathrm{r}$.

Cowling and Cray appeal to the modal distinction often made about numbers: numbers exist at all possible worlds, but don't exist in any of those worlds. To exist in a world is to occupy some region of that world. Swapping talk of worlds for talk of spatiotemporal regions, we can then say that $\mathrm{x}$ exists in a region $r$ if it occupies $r$, and that if $x$ occupies any region then it is concrete. Abstracta, by contrast, are those entities that exist at regions without occupying them, just as numbers supposedly exist at worlds without existing in them.

The thought is that we can then say that abstract entities, in virtue of existing at spacetime regions, rather than in them, can be causes and effects, since this is compatible with (P1) and (P2). Instead, this rejects (P3) by claiming that abstracta are located after all, they just don't occupy the regions at which they're located. This is supposed to be compatible with Relativity.

There are a number of difficulties with this proposal. First, note that it only provides a necessary condition for being abstract. As Cowling and Cray recognize, their account of omnipresence applies to the Abrahamic god, the Dao, and perhaps the Brahman (which was their primary motivation), but such entities, if they exist, are often taken to be concrete. It may also apply to mental entities, if we wanted to say that they are located at the regions occupied by the physical brains they depend on. It's not clear what else could be added to the Way of Occupation 
so that it provides jointly necessary and sufficient conditions. Necessary existence won't help since deities are thought to exist necessarily, too, nor would this help with the causal efficacy of putatively contingent abstracta such as fictional characters and musical works.

Second, the abstract/concrete distinction is usually thought to be non-relational, i.e. an entity is abstract simpliciter, not in virtue of the relations it stands in to regions of spacetime or possible worlds (see Cowling 2017, pp. 70-2 for discussion). The standard Way of Negation is monadic: an entity doesn't lack a spacetime location in virtue of anything else.

Third, it relies on the alleged distinction between existing 'at' and existing 'in', but it's not at all clear that this tracks a substantial metaphysical divide. While occupying a region is a perfectly understandable notion - presumably that's what all physical objects do - existing at a region without occupying it doesn't give any idea of what this is supposed to be. Cowling and Cray take the distinction to be intelligible, but they offer nothing by way of clarifying remarks except the analogy with numbers existing 'at' worlds. The slight linguistic difference between the two prepositions doesn't seem to track any obvious or substantial metaphysical distinction. Indeed, if we were conversing in a language without distinct terms for these prepositions, then we wouldn't even be able to formulate the position.

Finally, notice that the argument from Relativity can be rewritten in terms of occupying regions without having to change anything else I've said. For example, (P1) can be rephrased as follows:

$\left(\mathrm{P}^{*}\right)$ If $\mathrm{x}$ is causally efficacious, then $\mathrm{x}$ occupies a temporal region.

The other premises can be reformulated accordingly, and this would be perfectly compatible with the Way of Occupation while still making the same point. Therefore, I think we should reject the 
Way of Occupation, or at least remain agnostic about its potential until a fully reductive analysis is given.

\section{Conclusion}

I've argued that causation requires its relata to have temporal location, and that abstract objects cannot have temporal location without spatial location - they either have or lack spatiotemporal location. One can't give up temporal location without also giving up causal efficacy, but neither can one retain both temporal location and the non-spatial criterion. Abandoning the non-spatial criterion is highly undesirable: it appears to be a necessary condition for being abstract, and we would lose hold of what distinguishes abstract objects like numbers and properties from concrete objects like wombats and capybaras. The standard view of abstract objects is that they aren't located, since it seems prima facie odd to ask where abstract entities like the number two or Sherlock Holmes are. As a result, we are forced to accept the full-blown Way of Negation, an entity is abstract if and only if it lacks a spatiotemporal location, from which it follows that abstract objects are causally inert. If something is causally efficacious, then it cannot be abstract, and thus we have a principled argument for the causal inertness of abstract objects.

Notice what this argument does not do: it doesn't establish the causal inertness of abstracta in all possible worlds. Worlds with a Newtonian spacetime are worlds where an object can have temporal location but no spatial location, and thus abstract objects can have temporal location and causal efficacy without spatial location. The argument from Relativity only secures their causal inertness in the actual world and other nomically possible worlds. A further argument would be needed for the claim that they are causally inert across all possible worlds. Nonetheless, if we can't secure the causal efficacy of abstract objects in the actual world, then I doubt the position is of any real interest. The argument from Relativity relies on the standard way of characterizing abstract 
objects, so one could adopt an alternative account of being abstract that doesn't appeal to a lack of spatial or temporal location (but see Brock, Maslen, and Ngai (2013, pp. 64-8) for difficulties with various other ways if one wants to adopt causal efficacy). But at least on the standard view, abstracta are causally inert. $^{25}$

If the argument of this paper is correct, then non-causal solutions to the problems of reference, knowledge, and artistic practice would need to be given, but this isn't an insuperable difficulty. Linsky and Zalta (1995) defend a descriptions theory of reference for abstracta. We could adopt an a priori account of knowledge for abstract entities (cf. Steiner 1973 and Brown 2012). In the case of artistic practice, we may just need to slightly revise or re-describe our practices. For example, the act of creation of fictional characters or musical works can be construed non-causally, as Thomasson (1999) has argued, or understood as creative discovery, as Dodd (2007) has suggested. But these are problems for another paper.

Tim Juvshik University of Massachusetts Amherst

Acknowledgements: Thanks to Phil Bricker, Wesley Cray, Maya Eddon, David Friedell, Dan Korman, Chris Meacham, Josh Mozersky, two anonymous referees, and audiences in Kansas City, Pittsburgh, Toronto, Budapest, and San Diego for comments and criticisms which greatly improved this paper.

\section{Notes}

${ }^{1}$ Azzouni (2008) is an interesting exception.

${ }^{2}$ Lewis (1986), Cresswell (2010) and Cowling (2017) all entertain the idea but aren't committed to it.

${ }^{3}$ See Kripke (1981) and Putnam (1975). Thomasson (1999) adopts a hybrid theory of reference to deal with this problem.

${ }^{4}$ See Benacerraf (1983) for the initial version of the worry, but see Field (1989) for a reliabilist version. See also Steiner (1973), Callard (2007), Liggins (2010), Cresswell (2010), and Brown (2012) for discussion.

5 See Thomasson (1999), Caplan and Matheson (2004), Dodd (2007, 2012), Kania (2012), Rossberg (2012), and Brock, Maslen and Ngai (2013) for discussion of this motivation. 
${ }^{6}$ Thomasson (1999, pp. 38) also appears to adopt the lack of spatial location as a criterion for being abstract while accepting that, at least for fictional characters, they are created and exist at particular times. However, she elsewhere (1999, pp. 126) appears to characterize abstract entities as lacking a spatiotemporal location and being causally inert.

${ }^{7}$ The argument from Relativity is recognized as a problem by Brock, Maslen, and Ngai (2013, pp. 81 ) but they leave it undeveloped; this paper rectifies that.

${ }^{8}$ See Paul (2000) for a defense of property-causation and Whittle (2016) for a defense of objectcausation. Mellor (2004) defends facts as the primary causal relata, but takes facts to be spatiotemporally located, which is compatible with my argument. Alternatively, many people take facts to be abstract entities, in which case all causation would be between abstracta, which is intuitively implausible.

${ }^{9}$ I take the related notion of causal powers to be more or less interchangeable with causal efficacy or being a cause. See Whittle (2016) and Buckareff (2017) for discussion.

${ }^{10}$ See Burgess and Rosen (1997, pp. 16-25) for further discussion of Lewis' ways, and see Cowling (2017, ch. 2) for a recent discussion of various other ways one could make the distinction.

${ }^{11}$ See Lewis (1986, pp. 83ff.) for discussion.

12 It's accepted by, for example, Wolterstorff (1970), Dummett (1973), Wright (1983) Benacerraf (1983), Hale (1987), Maddy (1990), Linsky and Zalta (1995), Lowe (1995), Balaguer (1998), and Katz (1998), to name a few.

${ }^{13}$ It is also sometimes suggested that immanent universals and tropes are counterexamples to the Way of Negation, since they are taken to exist wherever they're instantiated. However, those who defend the existence of such entities explicitly take them not to be abstract. For example, Armstrong (1989, pp. 77) argues that immanent universals are constituents of objects and thus are not denizens of some otherworldly heaven. Thus, it's a mistake to think of such entities as abstract. 14 The distinction is also thought to be exhaustive, i.e. everything that exists is either abstract or concrete.

${ }^{15}$ While both Katz (1998, pp. 138ff.) and Williamson (2013, pp. 7n9) reject exclusiveness for impure sets, they do so because it conflicts with their broader metaphysical theories about, e.g. existence or modality.

${ }^{16}$ By contrast, Lewis (1991, pp. 32-33) argues that there's equal reason to think that impure sets are located as unlocated, so professes not to know which view to adopt.

${ }^{17}$ Katz (1998, pp. 125) and Dowe (2009, pp. 30ff.) also make this point.

${ }^{18}$ Minkowski spacetime, the spacetime of Special Relativity, is a special case of a Lorentzian spacetime. Minkowski spacetime generally refers to flat hyperbolic spacetimes, whereas Lorentzian spacetimes include curved hyperbolic spacetimes.

${ }^{19}$ See Maudlin (2012, ch. 4) for discussion.

${ }^{20}$ See Lewis (1973).

${ }^{21}$ Michael Dummett (1973, pp. 473) takes the vacuous satisfaction of counterfactual conditionals by abstracta to be a prima facie reason in favour of their causal inertness. Callard (2007, pp.352n6) also considers this argument.

22 This may have been Plato's view, although there's significant debate about how to understand his remarks, especially in the Phaedo. See Vlastos (1969) for general discussion of this issue; he argues that Plato's view on instantiation is best understood as a kind of tautological explanation for why objects instantiate the properties they do.

${ }^{23}$ James Robert Brown (2012) suggests this in the case of mathematical entities. 
${ }^{24}$ Lewis (1973, pp. 562 and passim) introduces the distinctness condition on causation to exclude cases of non-causal dependence. Distinctness is usually characterized as (1) non-identity, (2) nonoverlapping, and (3) neither is entailed by the other. It's on the last criterion that universals seem to flounder, since if we assume that there are Platonic universals, then any concrete instance of a universal entails that there is the corresponding abstracta.

${ }^{25}$ There is a quick argument from Alexander's dictum/the Eleatic Principle and the argument from Relativity to show that abstract objects don't exist. That is, if everything that exists must make a causal contribution to the world, and we have a principled argument against the causal efficacy of abstracta, then it follows that there are no abstract entities. However, since the argument from Relativity is restricted to nomically possible worlds, it doesn't rule out the causal efficacy of abstracta in nomically impossible worlds, so it may be possible that abstracta are causally efficacious in a broader sense, which may be sufficient to sidestep such an argument.

\section{References}

Armstrong, David. 1989. Universals: An Opinionated Introduction (Boulder, CO: Westview Press).

Azzouni, Jody. 2008. "A Cause for Concern: Standard Abstracta and Causation,” Philosophia Mathematica vol. 3, no. 16, pp. 397-401.

Balaguer, Mark. 1998. Platonism and Anti-Platonism in Mathematics (Oxford: Oxford University Press).

Baron, Sam and Kristie Miller. 2015. "Causation sans Time," American Philosophical Quarterly, vol. 52, no. 1, pp. 27-40.

Benacerraf, Paul. 1983. "Mathematical Truth," in Philosophy of Mathematics (2nd edition), ed. Paul Benacerraf and Hilary Putnam (Cambridge: Cambridge University Press), pp. 403420 .

Black, Max. 1971. "The Elusiveness of Sets," Review of Metaphysics, vol. 24, no. 4, pp. 614636.

Brock, Stuart, Cei Maslen and Justin Ngai. 2013. "A Puzzle About Fictional Characters,” in From Fictionalism to Realism, ed. Carola Barbero, Maurizio Ferraris, and Alberto Voltolini (Newcastle: Cambridge Scholars), pp. 63-86.

Brown, James Robert. 2012. Platonism, Naturalism, and Mathematical Knowledge (New York: Routledge).

Buckareff, Andrei. 2017. “A Critique of Substance Causation,” Philosophia, vol. 45, no. 3, pp. 1019-1026.

Burgess, John and Gideon Rosen. 1997. A Subject with No Object: Strategies for Nominalistic Interpretation of Mathematics (Oxford: Oxford University Press). 
Callard, Benjamin. 2007. "The Conceivability of Platonism,” Philosophia Mathematica, vol. 3, no. 15 , pp. $347-356$.

Caplan, Ben and Carl Matheson. 2004. “Can a Musical Work be Created?” British Journal of Aesthetics, vol. 44, no. 2, pp. 113-134.

Cowling, Sam. 2017. Abstract Entities (New York: Routledge).

Cowling, Sam and Wesley Cray. 2017. "How to Be Omnipresent," American Philosophical Quarterly, vol. 54, no. 3, pp. 223-234.

Cresswell, Max J. 2010. “Abstract Entities in the Causal Order,” Theoria, vol. 76, pp. 249-265.

Dodd, Julian. 2007. Works of Music: An Essay in Ontology (Oxford: Oxford University Press).

----- 2012. "Defending the Discovery Model in the Ontology of Art: A Reply to Amie

Thomasson on the Qua Problem," British Journal of Aesthetics, vol. 52, no. 1, pp. 75-95

Dowe, Phil. 2009. “Absences, Possible Causation, and the Problem of Non-Locality," The Monist, vol. 92, no. 1, pp. 23-40.

Dummett, Michael. 1973. Frege: Philosophy of Language (London: Duckworth).

Field, Hartry. 1989. Realism, Mathematics and Modality (Oxford: Blackwell).

Hale, Bob. 1987. Abstract Objects (Cambridge: Blackwell).

Juvshik, Tim. 2018. “Abstract Objects, Causal Efficacy, and Causal Exclusion,” Erkenntnis, vol. 83 , no. 4, pp. 805-827.

Kania, Andrew. 2012. "Platonism vs. Nominalism in Contemporary Musical Ontology," in Art and Abstract Objects, ed. Christy Mag Uidhir (Oxford: Oxford University Press), pp. 197219.

Katz, Jerrold. 1998. Realistic Rationalism (Cambridge, MA: Bradford Books).

Kripke, Saul. 1981. Naming and Necessity (Oxford: Blackwell Publishers).

Lewis, David. 1973. “Causation,” Journal of Philosophy, vol. 70, pp. 556-567. 1986. On the Plurality of Worlds (Malden, MA: Blackwell Publishing).

------ 1991. Parts of Classes (Oxford: Basil Blackwell).

Liggins, David. 2010. “Epistemological Objections to Platonism,” Philosophy Compass, vol. 5, no. 1, pp. 67-77. 
Linsky, Bernard and Edward N. Zalta. 1995. "Naturalized Platonism vs. Platonized Naturalism," Journal of Philosophy, vol. 92, no. 10, pp. 525-555.

Lockwood, Michael. 1989. Mind, Brain and the Quantum: The Compound 'I' (Cambridge, MA: Basil Blackwell).

Lowe, E. J. 1995. “The Metaphysics of Abstract Objects," Journal of Philosophy, vol. 92, no. 10, pp. 509-524.

Maddy, Penelope. 1990. Realism in Mathematics (Oxford: Clarendon Press).

Maudlin, Tim. 2012. Philosophy of Physics: Space and Time (Princeton: Princeton University Press).

Mellor, D. H. 2004. "For Facts as Causes and Effects," in Causation and Counterfactuals, ed. John Collins, Ned Hall, and L. A. Paul (Cambridge, MA: MIT Press), pp. 309-324.

Paul, L. A. 2000. “Aspect Causation,” Journal of Philosophy, vol. 97, no. 4, pp. 223-234.

Putnam, Hilary. 1975. "The Meaning of 'Meaning,"' in Mind, Language, and Reality: Philosophical Papers Volume 2, (Cambridge: Cambridge University Press), pp. 215-271.

Rossberg, Marcus. 2012. "Destroying Artworks," in Art and Abstract Objects, ed. Christy Mag Uidhir (Oxford: Oxford University Press), pp. 62-83.

Steiner, Mark. 1973. "Platonism and the Causal Theory of Knowledge," Journal of Philosophy, vol. 70, no. 3, pp. 57-66.

Thomasson, Amie. 1999. Fiction and Metaphysics (Cambridge: Cambridge University Press).

Vlastos, Gregory. 1969. "Reasons and Causes in the Phaedo," Philosophical Review, vol. 78, no. 3, pp. 291-325.

Weingard, Robert. 1977. "Relativity and the Spatiality of Mental Events," Philosophical Studies, vol. 31, no. 4, pp. 279-284.

Whittle, Ann. 2016. "A Defense of Substance Causation," Journal of the American Philosophical Association, DOI: 10.1017/apa.2016.1, pp. 1-20.

Williamson, Timothy. 2013. Modal Logic as Metaphysics (Oxford: Oxford University Press).

Wolterstorff, Nicholas. 1970. On Universals: An Essay in Ontology (Chicago: University of Chicago Press).

Wright, Crispin. 1983. Frege's Conception of Numbers as Objects (Aberdeen: University Press). 